\title{
Die dritte Welle
}

\author{
Norbert Lossau
}

\begin{abstract}
Erstmals haben Wissenschaftler durch die Messung von Gravitationswellen sehr wahrscheinlich das Verschmelzen eines schwarzen Loches mit einem Neutronenstern beobachten können. Eine Physikerin in Potsdam hat wichtige Grundlagen zu diesem wissenschaftlichen Erfolg beigetragen.
\end{abstract}

Am 25. April 2019 registrierten zwei Detektoren für Gravitationswellen um 10:18:26 Uhr MESZ ein Signal, das aufgrund seiner Form ganz eindeutig bei der Fusion von zwei Neutronensternen, rund 500 Millionen Lichtjahre von uns entfernt, entstanden sein muss. Die Gravitationswelle wurde vom Ligo-Detektor in Livingston im US-Bundesstaat Louisiana und vom Virgo-Detektor in Italien registriert. Der zweite Ligo-Detektor in Hanford im US-Bundesstaat Washington war zu diesem Zeitpunkt aus technischen Gründen außer Betrieb. Doch zwei Signale reichen für den Beweis, dass es sich nicht um zufälliges Rauschen gehandelt haben kann.

Viele Gravitationswellenforscher hatten sich just an diesem Tage auf einem Fachkongress („Enabling MultiMessenger Astrophysics“ - EMMA) in Baltimore versammelt, als sie von der Messung erfuhren. Es war ja erst das zweite Mal in der Geschichte der Gravitationswellenforschung, dass die Fusion von zwei Neutronensternen beobachtet werden konnte. Die Aufregung über diesen Erfolg brachte die ganze Tagung durcheinander. Ein Teilnehmer, der Physiker Andy Howell, twitterte: „Wir verlieren alle unseren Verstand hier auf der \#EMMA2019.“

Doch es kam noch besser. Nur einen Tag später, am 26. April 2019 um 17:22:17 Uhr MESZ schlugen die Gravitationswellendetektoren wieder an - dieses Mal alle drei Systeme, also beide Ligo-Detektoren und Virgo. Was die Forscher sahen, war eine Kurve, die deutlich anders aussah als alle bisher gemessenen. Noch steht der letzte Beweis aus, doch die Ligo-Forscher sind sich bereits ziemlich sicher, dass dieses Signal von der Fusion eines Neutronensterns mit einem schwarzen Loch verursacht wurde. Das wäre etwas völlig Neues. Gehofft hatten die Astrophysiker schon lange, dass der Nachweis solcher Vorgänge im Universum mithilfe von Gravitationswellenmessungen gelingen möge. Jetzt könnte es endlich so weit sein. Vorerst bleibt jedoch ein kleines "Restrisiko", dass es sich auch hier um die Fusion von zwei Neutronensternen gehandelt hat. In jedem Fall hat sich das kosmische Ereignis rund 1,2 Milliarden Lichtjahre von uns entfernt zugetragen. „Ich denke, die Klassifikation wird auf eine Fusion von schwarzem Loch und Neutronenstern hinauslaufen", prognostiziert der Physiker Chad Hanna von der Pennsylvania State University und Mitglied des LigoTeams für Datenanalyse.

So oder so ist der Doppelschlag vom 25. und 26. April ein großer Erfolg für die Gravitationswellenforschung. Der Umbau der Ligo-Detektoren, bei dem die Empfindlichkeit des Messsystems verbessert wurde, hat sich offenbar gelohnt. Nach einer längeren Wartungs- und Umbaupause waren die beiden Ligo-Detektoren erst am 1. April 2019 wieder in Betrieb gegangen. Seitdem haben sie auch schon wieder mehrere Fusionen von jeweils zwei schwarzen Löchern beobachten können. Das gehört quasi schon zur Routine der Gravitationswellenforscher, die im Mittel mit einem solchen kosmischen Ereignis pro Woche rechnen.

Ob die von einem Gravitationswellendetektor gemessenen Wellen von zwei schwarzen Löchern, zwei Neutronensternen oder der Fusion eines schwarzen Loches mit einem Neutronenstern stammen, lässt sich aus der genauen Form der Welle ermitteln. Eine weltweit führende Wissenschaftlerin für die Interpretation von Gravitationswellenformen ist Alessandra Buonanno, Direktorin am Potsdamer MaxPlanck-Institut für Gravitationsphysik. Mit Kreide zeichnet Alessandra Buonanno schwungvoll und ohne abzusetzen eine ästhetisch perfekte Wellenkurve an die große Wandtafel in ihrem Büro: „So sieht eine Gravitationswelle aus, wie sie beim Verschmelzen von zwei Neutronensternen entsteht." Jahrzehntelang hat sich die in Italien geborene Forscherin damit beschäftigt, welche Form Gravitationswellen im Detail haben - abhängig davon, bei welchem kosmischen Vorgang sie entstanden sind.

Dabei gilt es, konkrete Lösungen für eine sehr komplizierte, von Albert Einstein 1915 formulierte mathematische Gleichung zu finden. Diese sogenannte Einstein-Gleichung ist der mathematische Wegweiser dafür, dass es im Universum so etwas wie Gravitationswellen geben sollte. Einstein selbst hatte nicht geglaubt, dass es jemals gelingen würde, diese von ihm vorhergesagten Verzerrungen des kosmischen Raum-Zeit-Gefüges zu registrieren.

Bis vor vier Jahren konnte Alessandra Buonanno nicht wissen, ob ihre aufwendigen Berechnungen mit Papier und Bleistift jemals eine praktische Relevanz haben würden. Doch dann glückte 2015 in den USA mit den beiden LigoDetektoren erstmals der experimentelle Nachweis von Gravitationswellen - genau 1 oo Jahre nach der theoretischen Vorhersage durch Albert Einstein. „Da wusste ich dann, dass ich mich als Forscherin für die richtige Sache entschieden hatte", sagt Alessandra Buonanno.

Dass die Gravitationswellenforscher im Jahr 2015 und bei späteren Messungen aus den registrierten Signalen sofort ableiten konnten, auf welche Weise diese Wellen entstanden sein mussten - das ist insbesondere das Verdienst von Alessandra Buonanno. Nur weil sie vorab Hunderttausende von möglichen Wellenformen berechnet hat, kann man schnell in der Datenbank nachschauen, was genau diese Wellenform verursacht haben musste. Das genaue Aus- 
sehen der Gravitationswelle hängt unter anderem von den Massen der schwarzen Löcher ab. Die 2015 von den LigoDetektoren gemessene Wellenform passte genau zu jener Kurve in der Datenbank, die man für die Fusion von zwei schwarzen Löchern mit 29 und 36 Sonnenmassen erwartete. Ohne die Vorarbeit der Theoretiker hätten die Experimentalphysiker also gar nicht wissen können, was sie da eigentlich gemessen haben. Wie so oft in der Physik gilt auch hier: Man kann nur etwas finden, wenn man weiß, wonach man sucht. „Allerdings ist es auch denkbar, dass man im Experiment ein Signal sieht, das man bis dahin noch gar nicht kannte", sagt Buonanno, „dann hat man offenbar etwas Neues entdeckt. Und die Theoretiker sind gefordert, das noch Unbekannte zu beschreiben und zu erklären." Eigentlich hoffen Forscher immer, dass sie auch Dinge entdecken, von denen sie nicht wussten.

Der Ursprung der jetzt von den Ligo-Forschern registrierten Wellen ließ sich im Fall der verschmelzenden Neutronensterne ebenfalls sofort mit vorproduzierten „Templets“ erklären. Diese Steckbriefe für Signalformen verrieten, dass am 25. April ein solches Ereignis beobachtet wurde. Das einen Tag später aufgefangene Signal war nicht besonders intensiv, sodass die Interpretation zunächst schwieriger war. Die Forscher hoffen, dass die Welle tatsächlich von der Fusion eines schwarzen Loches mit einem Neutronenstern stammt.

"Auf genau so ein Ereignis haben wir bis jetzt gewartet", sagt Buonanno. Denn bisher ließen sich fast alle registrierten Gravitationswellen auf fusionierende schwarze Löcher zurückführen. Nur in einem Fall hatten zwei verschmelzende Neutronensterne die wellenförmigen Erschütterungen im Raum-Zeit-Kontinuum verursacht. Dieses Ereignis registrierten die Ligo-Detektoren am 17. August $2017 \mathrm{um}$ 14:41:04 Uhr MESZ. Es war damals der Ligo-Detektor im amerikanischen Hanford, der das erste Signal auffing. Kurz darauf traf dann die Gravitationswelle GW17081 7 auf den zweiten Ligo-Detektor in Livingston.

Buonanno ist fasziniert von der Präzision und Macht der Mathematik, die es ermöglicht, Phänomene in der realen Welt vorherzusagen und das Universum zu verstehen. "Ich habe bereits in der Schule Mathematik geliebt", erinnert sich die Forscherin, „doch die Leidenschaft für Physik war letztlich noch größer." In den Jahren vor der Matura hatte sie zwar noch erwogen, Medizin zu studieren, doch dann gab letztlich ein Zeitungsartikel den Ausschlag für die Physik. Die Nachricht von der Entdeckung eines neuen Elementarteilchens, des W-Bosons, am europäischen Forschungszentrum Cern in Genf faszinierte damals die junge Frau so sehr, dass sie ein Physikstudium an der Universität Pisa begann. Mit Schwerpunkt Teilchenphysik.

In ihrer Doktorarbeit beschäftigte sich Alessandra Buonanno mit der Kosmologie des frühen Universums. „Da ging es um Stringtheorie und kosmische Inflation“, erklärt die Physikerin. Nach einem neunmonatigen Forschungsaufenthalt am Cern führte sie ihr Weg zum Institute des Hautes Études Scientifiques, das rund 3o Kilometer südwestlich von Paris liegt. Dort kam sie zum ersten Mal mit der Theorie von den "Fuzzy Templets“ in Kontakt - einer Methode, mit der sich Wellenformen ohne Computer von Hand berechnen lassen - nicht exakt, aber doch näherungsweise (fuzzy) mit einem Fehler von ungefähr zehn Prozent.

„Grundlage für meine gesamte spätere Forschungsarbeit war ein 1998 erschienenes Paper des südafrikanischen Physikers Frans Pretorius", erinnert sich Buonanno. Fortan berechnete sie nach diesem Fuzzy-Rezept die Formen Tausender von Wellenformen, von denen sie noch immer schwärmerisch sagt, sie seien von großer Schönheit. Sie vergleicht Physik sogar mit Kunst - mit dem Malen oder Musizieren. Und sie kann das beurteilen, denn als Teenager spielte sie leidenschaftlich Klavier. Die in Potsdam errechneten, wirklich schön anzusehenden Gravitationswellen haben es sogar zum Briefmarkenmotiv geschafft. Anlässlich der Entdeckung von Gravitationswellen gab die Deutsche Post eine $7^{0}$-Cent-Sonderbriefmarke heraus. Eine stark vergrößerte Kopie dieser Marke schmückt das Büro von Alessandra Buonanno.

"Auch die Einstein-Gleichung ist einfach sehr schön", sagt die Physikerin. Doch leider eben auch so kompliziert, dass Computer für die exakte numerische Berechnung einer Wellenform Tage benötigen. Viel schneller lassen sich die analytischen Näherungslösungen gewinnen. Und genau darin ist Buonanno quasi Weltmeisterin. In den Datenbanken der Gravitationswellenforscher stehen heute mehr als 250000 solcher Templets für das Verschmelzen von zwei schwarzen Löchern sowie die Fusion eines Neutronensterns mit einem schwarzen Loch zur Verfügung. Die Wellenformen für zwei verschmelzende Neutronensterne sind schwieriger zu ermitteln. Hier liegen erst 50000 „Fingerabdrücke“ bereit.

Ist erst einmal ein kosmisches Ereignis durch die Messung von Gravitationswellen und den Vergleich mit Templets identifiziert worden, lohnt natürlich in einem solchen Fall im nächsten Schritt die präzise Simulation der gemessenen Wellenform in einem Supercomputer. Dann lassen sich aus den Messdaten nachträglich nicht nur die genauen Massen der fusionierten Himmelsobjekte ermitteln, sondern auch deren Durchmesser und die Rotationsgeschwindigkeiten. Alle diese Parameter haben einen Einfluss auf die konkrete Form der Gravitationswellen.

Zwischen 2002 und 2014 arbeitete Buonanno mehrfach als Gastforscherin am California Institute of Technology (Caltech). Im Jahr 2005 wurde sie Professorin für Physik an der University of Maryland in College Park. Und 2014, ein Jahr vor der erstmaligen Messung von Gravitationswellen, wurde sie Direktorin am Max-Planck-Institut für Gravitationsphysik in Potsdam. Außerdem ist sie seit 2017 auch Honorarprofessorin an der Berliner Humboldt-Universität sowie der Universität Potsdam.

Mit Gravitationswellen könnten sich viele weitere Phänomene im Universum erforschen lassen - zum Beispiel Supernova-Explosionen. „Auch Supernovae können ein kurzes Gravitationswellensignal von einigen zehn Millisekunden abstrahlen“, sagt Buonanno, „doch diese Signale sind so schwach, dass wir sie mit den heutigen Detektoren nur dann werden registrieren können, wenn sich eine Sternenexplosion in unserer kosmischen Nachbarschaft ereignet." 


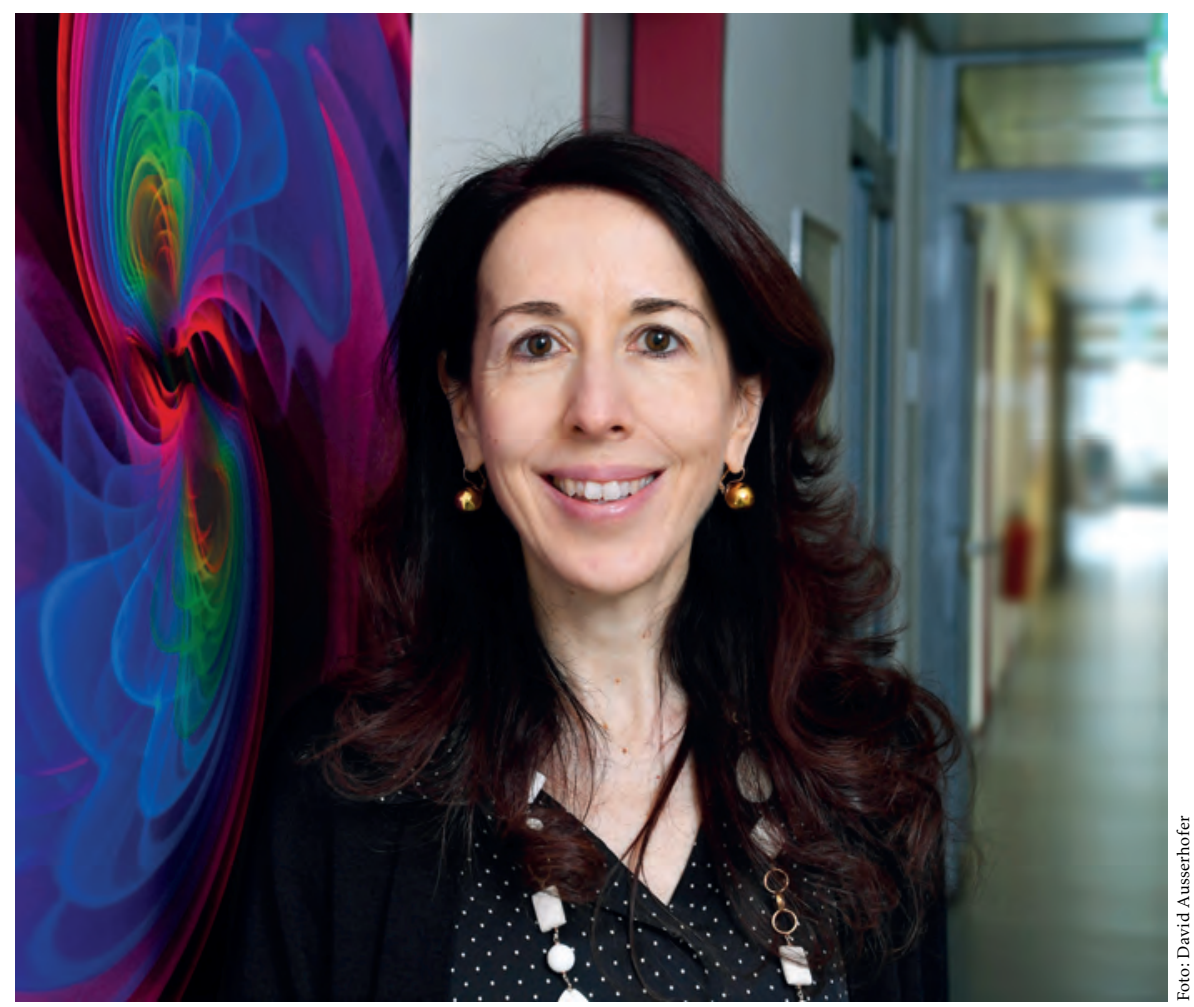

Alessandra Buonanno, Max-Planck-Institut für Gravitationsphysik

Zuletzt hatte es 1987 eine solche Supernova-Explosion gegeben. Wären damals schon die heutigen Detektoren für Gravitationswellen verfügbar gewesen, hätte man die Signale der Supernova „1987A“ auffangen können. Nach Berechnung von Astrophysikern kann man allerdings nur rund einmal pro Jahrhundert mit einer Supernova rechnen, die sich mit Gravitationswellendetektoren messen lässt.

„Auch Pulsare können Gravitationswellen aussenden“, sagt Buonanno, „vorausgesetzt sie haben einen Berg, der dem Himmelskörper eine Asymmetrie verleiht." Besonders spannend dürfte indes die Erforschung des Urknalls mithilfe von Gravitationswellen sein. Es gibt mehrere kosmologische Theorien dazu, wie sich das frühe Universum entwickelt hat. Durch die Messung von Gravitationswellen in bestimmten Wellenlängenbereichen ließe sich endlich entscheiden, welche dieser Theorien die richtige ist.

Auf die Klärung dieser Frage werden die Astrophysiker allerdings noch bis Mitte des Jahrhunderts warten müssen. Dann sollen im Rahmen des Esa-Programmes „Voyage 2050" Satelliten ins All gebracht werden, die solche Messungen ermöglichen. Mit den bereits für die 203oerJahre geplanten Lisa-Satelliten können die von der Ausdehnung des Universums kurz nach dem Urknall („,kosmische Inflation") verursachten Gravitationswellen noch nicht beobachtet werden. „Dafür ist die Messempfindlich- keit dieses Gravitationswellendetektors zu klein“, sagt Buonanno.

Die Formen von Gravitationswellen werden indes nicht nur in Potsdam errechnet. Auch in Paris, am Caltech, an den US-Universitäten Cornell und Rochester sowie der britischen University of Cardiff sind Wissenschaftler damit befasst. Ein Alleinstellungsmerkmal des Potsdamer Max-Planck-Instituts ist jedoch, dass dort sowohl „FuzzyTemplets" berechnet als auch exakte Computersimulationen durchgeführt werden. Anderenorts konzentriert man sich auf das eine oder andere.

Wissenschaft, insbesondere die global stark vernetzte Gravitationswellenforschung, basiert auf der Kooperation von vielen. „Man braucht Teams“, kommentiert Buonanno, „man braucht aber auch unabhängige Denker.“ Buonanno, die sowohl die italienische als auch die US-amerikanische Staatsbürgerschaft besitzt, fühlt sich als Weltenbürgerin. Wer sich mit Phänomenen beschäftigt, die sich in den unvorstellbaren Weiten des Alls abspielen, dem müssen irdische Ländergrenzen wohl kleinkariert vorkommen. „Ich habe viele verschiedene Kulturen kennengelernt und davon sehr profitiert", sagt Buonanno, die eine leidenschaftliche Fotografin ist, gleichermaßen klassische Musik, Jazz und Blues liebt und sich gern gute Filme im Kino anschaut. Doch in einem Punkt ist sie immer ihrer Heimat verbunden geblieben: „Wenn ich zu Hause koche, dann koche ich italienisch.“ 